\title{
Assessment of the dynamics of bone edema in fractures of long tubular bones in children in the process of osteoregeneration according to magnetic resonance imaging
}

\begin{abstract}
Children 6-10 years old are distinguished by special mobility, carelessness, often neglect of danger and constitute the main concern of children's traumatologists. Excessive reduction in the timing of immobilization of the limb is not always justified. In the MRI study, after manual repositioning and gypsum immobilization, a wave-like increase in signal intensity occurs, reaching a maximum value on the 20th day after the trauma, with further decrease. The exception is a group of children of primary school age who have a high signal intensity for 40-45 days. MRI examination allows to assess the degree of severity of trabecular edema, the development of granulation tissue and to predict the development of bone callus without radiation.
\end{abstract}

Volume 5 Issue 3 - 2018

\author{
Tarasenko LL, Tarasenko TS \\ University Surgut state University, Russia
}

Correspondence: Tarasenko LL, University Surgut state University, Surgut, Nefteyugansk highway street 20, Russia, Tel +79226529729, Email lyubtarasenko@tandex.ru

Received: April 20, 2016 | Published: June 14, 2018

Keywords: children, traumatologists, MRI, trabecular edema, granulation tissue

\section{Introduction}

Children 6-10 years old very active, incautious, careless of danger this is a main care children's traumatologist. Excessive shortcut immobilization extremity is not for all time correctly. After manual reposition and plaster immobilization MRI show wavy rise in intensity of the signal and reach the maximum values on the $20^{\text {th }}$ day after the trauma with the continued its decrease. The exception is a group of children, the younger school age, who save high intensity signal during the 40-45-th days. MRI allows an estimate of the degree of expression of the trabecular edema, the development of granulation, and the development of the callus without X-ray.

Among the causes of childhood injuries in the first place is a domestic trauma, which, according to many authors, is about $70 \%{ }^{1}$ Basically, it falls during games and pranks, jumping and falling from a height. Children 6-10 years old are distinguished by special mobility, carelessness, often neglect of danger and constitute the main concern of children's traumatologists

Diaphysis of long bones in young children are fractured more often than epiphyses, the cartilaginous cover of which, to certain limits, extinguishes the strength of the trauma, especially directed along the longitudinal axis of the segment. Regeneration processes for all these various injuries in children occur more intensively, the younger the child. ${ }^{1}$

Children's traumatology has characteristic scientific and practical features. High motor activity of children, uncontrolled behavior, especially after the abatement of pain, with conservative treatment of distal fractures of the limbs, does not allow rigid fixation of bone fragments, which in turn leads to micro-mobility, and, consequently, to the development of violation of osteoreparation. ${ }^{2}$ Excessive reduction of the limb immobilization time (up to 2-3 weeks), as indicated in some educational publications, is also not always justified. ${ }^{1}$

The growing interest in the problem of bone tissue repair is also associated with an increase in the number of complications that arise during the treatment of fractures, despite the use of modern therapies. In recent years, in children's practice, there has been a trend towards an extension of the timing of consolidation and an increase in incomplete osteogenesis in bone fractures.

To date, in the domestic and foreign literature there is a significant amount of work devoted to elucidating the characteristics of bone metabolism in the treatment of fractures by various methods of osteosynthesis. ${ }^{4,5}$ It seems obvious that to use repositions in practice, the trauma orthopedist should be armed with information about the bone reaction to trauma or reposition, which allows to control and predict the treatment process.

\section{Purpose of the study}

The aim of the study was to study and systematize the MRIsymptomatology of fracture healing in the near future of conservative treatment, comparing the dynamics of the MR signal depending on the stage of reparative osteogenesis and the severity of granulation tissue development.

\section{Materials and methods}

We examined 87 children admitted to a pediatric trauma center with fractures of long tubular bones of the forearm and lower leg. These selection criteria are due to the possibility of treating fractures of these bones with conservative methods of treatment, in particular by manual reposition and gypsum immobilization. Among them were 59 boys and 28 girls aged 4 to 13 years. The children were divided into two groups (the choice of the periods of life of children is based on the work), ${ }^{6}$ where the first period up to 6 years (the period of formation of milk teeth) is 25 people, then the younger school age from 7 to 12 years is 62 people. The greatest number of children (71.2\%) fell to the junior school age from 7 to 12 years. The fractures of the forearm bones were in 62 patients, the bones of the lower leg in 25 patients. Fractures with displacement in 57 patients, without displacement in 
30 patients. All children in the anamnesis had an indication of the presence of trauma: falling from the height of their own growth, falling to their feet, on their hands from any structures of various heights (slide, horizontal bar, building, tree), unsuccessful physical exercise in the lesson of physical education.

All patients underwent clinical, radiological and MRI studies of the damaged segment. The timing of the clinical trial was compared with the main stages of reparative osteogenesis and radiologic manifestations: the first day after the trauma, 7-10 days, 21-24 days, 40-45 days (immediately after the removal of immobilizing dressings). In a clinical study, a collection of complaints was made, which can be specific and specific only at an older age. Then crepitation, mobility of the damaged segment was determined palpation. All patients underwent manual repositioning, immobilization with plaster gypsum.

Radiography of the limbs was carried out with the Philips Tele Diagnost, Philips BuckyDiagnost, Philips Essenta RC in two mutually perpendicular planes (direct and lateral projections with the X-ray beam centering to the fracture region with the capture of a nearby joint). Magnetic resonance tomography was carried out on the apparatus with a magnetic field strength of $1.5 \mathrm{~T}$, using soft coils, scanning programs T1, PDW, T2-STIR in the axial, coronal and sagittal planes. Additionally, the pulse sequence GRE was used. ${ }^{7}$ The visualization of the bone marrow depends on the age of the patient. In the first years of life, when the bone marrow performs hematopoietic function, it has a high uniform signal intensity in the STIR regime. By the age of 14-16, the red bone marrow has replaced the yellow bone. This process is characterized by a multifocus decrease in signal intensity. ${ }^{8}$ In connection with these data, as well as for the differentiation of the signal from hemorrhage and edema, in addition, all patients were examined with a pulse sequence to suppress the signal from fat.

For the objectivity of signal analysis, the computer program Measurements Ellipse was used, which makes it possible to measure the intensity of a signal in conventional units. The area of the measured zone of interest was identical for all studies.

\section{Results}

\section{Features of fractures of the forearm bones of children of the first age group (up to 6 years) in 18 patients}

On the first day of the fracture of long tubular bones with MRI, a fracture line was defined, in the form of a violation of the integrity of the cortical layer with displacement and without displacement. In the magnetic resonance image, the fracture was characterized by a linear increase in the intensity of the signal from around the fracture line on T2-weighted images (VI) and a decrease in signal intensity on T1-VI of different widths.

When studying in the regime with suppression of the signal from fat, the width of the signal intensity increase around the fracture line was larger, the increase in signal intensity was moderate. These changes are due to the edema of the bone marrow and a hemorrhage into the bone marrow.

Also, in most cases, deformation of a long tubular bone was observed. In soft tissues, in the projection of the fracture line, a lenticular fluid accumulation with a rather distinct contour was determined, which was caused by hematomas and hemorrhages (damage to small vascular branches) in response to trauma. During MRI examination of the damaged segment after 7-10 days, a progressive increase in the intensity of the signal on the T2-STIR of a diffuse nature was determined and the area of its spread increased. The intensity of the signal decreased from the region of the fracture to the periphery. Intensity of the signal from the intermuscular hematoma did not change, although the structure became heterogeneous.

During MRI examination of the damaged segment, the maximum increase in signal intensity on T2-STIR was determined after 21-24 days. The volume of intermuscular hematoma decreased. When MRI examination of the damaged segment after 40-45 days, the severity of the high cigul (intensity of the trabecular edema) was decreased by 1.1-1.3 times.

\section{Features of fractures of the forearm bones of children of the second age group ( 6 to 13 years) in 44 patients}

On the first day of the fracture of long tubular bones in children 6 to 13 years of age, the MRI examination showed similar changes as in children of younger preschool age: the fracture line, as a linear increase in signal intensity from around the fracture line to T2-STIR weighted images (VI) and a decrease in the intensity of the signal on T1-VI of different widths. When studying in the regime with suppression of the signal from fat, the width of the signal intensity increase around the fracture line was larger, the increase in signal intensity was moderate. In soft tissues, in the projection of the fracture line, a lenticular fluid accumulation with a rather distinct contour was determined. The results of the research within 7-10 days from the moment of trauma coincided with the data of the children of the first age group in the form of a progressive increase in the intensity of the signal on the T2-STIR of a diffuse nature and an increase in the range of its distribution.

During MRI examination of the damaged segment, the maximum increase in signal intensity on T2-STIR was determined after 2124 days. The intensity of the signal for 120-152 units. was lower than in the first age group. The volume of intermuscular hematoma decreased. At MRI examination of the damaged segment in 40-45 days, a decrease in the severity of the high cigar was found to be 1.11.3 times.

\section{Features of fractures of the bones of the lower leg of children of the first age group (up to 6 years) in 7 patients}

The first day of fracture of the shin bones - in the MRI study, similar changes were detected, as in fractures of the forearm bones in the form of a violation of the integrity of the cortical layer with a linear increase in signal intensity from around the fracture line to T2-VI, T2STIR and a decrease in signal intensity at T1-VI of different widths. In soft tissues, in the projection of the fracture line, a lenticular fluid accumulation with a rather distinct contour was determined.

During MRI examination of the damaged segment after 7-10 days, a progressive increase in the intensity of the signal on the T2-STIR of a diffuse nature was determined and the area of its spread increased. The intensity of the signal decreased from the region of the fracture to the periphery. Intensity of the signal from the intermuscular hematoma did not change, although the structure became heterogeneous. During MRI examination of the damaged segment, the maximum increase in signal intensity on T2-STIR was determined after 21-24 days. The volume of intermuscular hematoma decreased. When MRI examination of the damaged segment after 40-45 days, the severity of the high cigul (intensity of the trabecular edema) was decreased by 1.1-1.3 times. 
Features of fractures of the bones of the lower leg of children of the second age group (6 to 13 years) in 18 patients

The first and second periods - in the MRI study, similar changes were detected as in the first group of children with fractures of the shin bones. Those. violation of the integrity of the cortical layer with a linear increase in signal intensity from around the fracture line to T2-
VI, T2-STIR and a decrease in signal intensity on T1-VI of different widths and a progressive increase in signal intensity by 7-10 days after trauma.

During MRI examination of the damaged segment, the maximum increase in signal intensity on T2-STIR was determined after 2124 days. The volume of intermuscular hematoma decreased. These changes were maintained until 40-45 days without dynamics to a decrease in signal intensity (Figure 1).

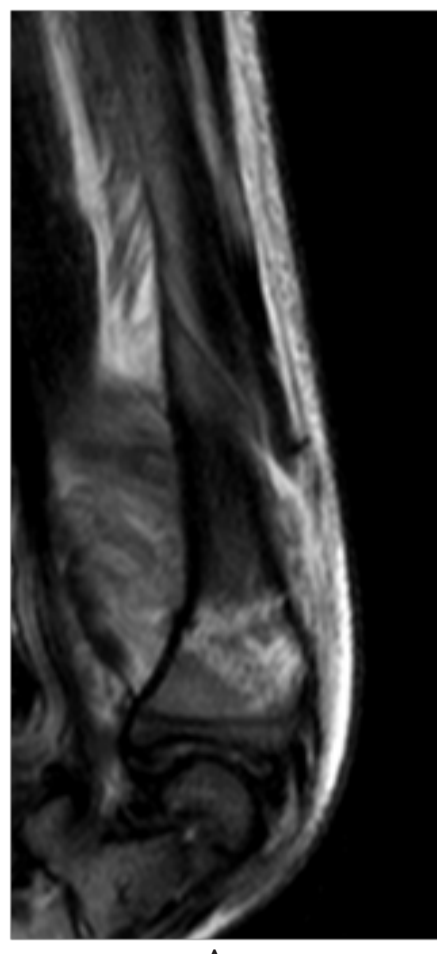

A

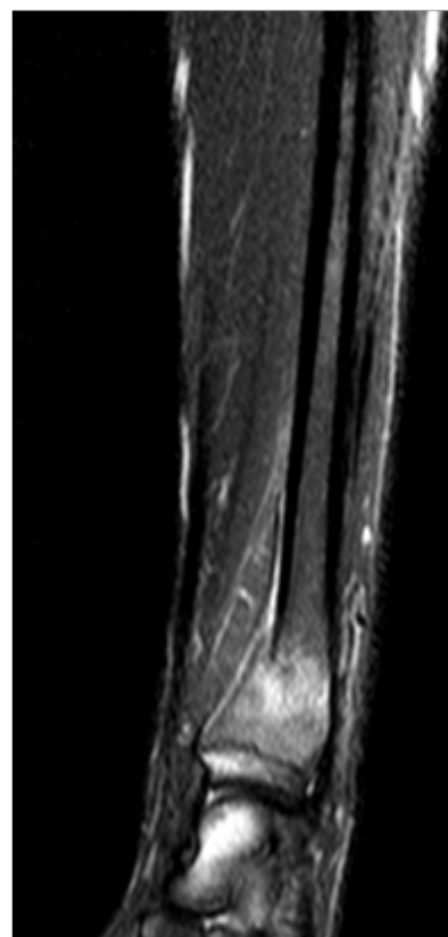

B

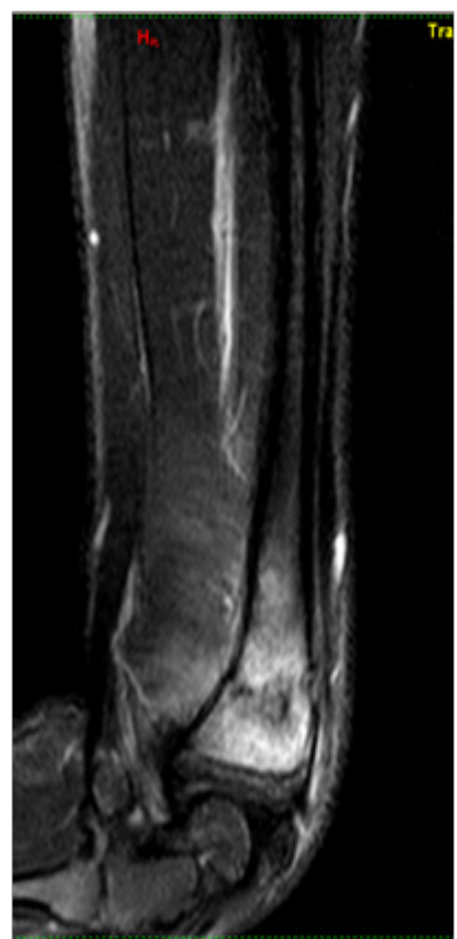

C

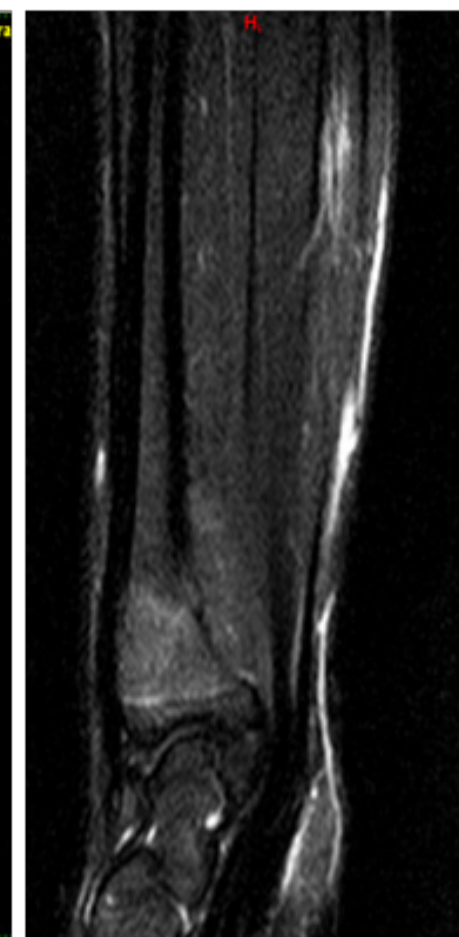

D

Figure I Patient H, I2 years. MRI-bones of the left forearm, pulse sequence T2-STIR. Fracture of the distal metadiaphysis of the radius with displacement and light palmar angular deformation;A.The first day after the injury and manual repository; B. $8^{\text {th }}$ day after injury; in the $23^{\text {rd }}$ day after injury; C. $40^{\text {th }}$ day after trauma and removal of the immobilizing bandage. D. An increase in the intensity of the signal around the fracture line is observed, reaching a maximum signal intensity on the $23^{\text {rd }}$ day after trauma.

\section{Discussion}

Regulation of osteogenesis in case of damage is performed by a complex set of factors, including mechanical conditions for the formation of a full-fledged regenerate, vascular responses, the effect of the neuroendocrine system, the effect of metabolites and growth factors. ${ }^{9}$ At the heart of the bone substance is a connective tissue that has a high plasticity. Between bone fragments with bone damage granulation tissue is formed, but the degree of its development is different. ${ }^{10}$

According to the literature, on the 1st day, between the fragments, a hematoma is formed, in many cases extensive, with deposition of fibrin and the presence of fragments of necrotic bone. There is more or less abundant leukocyte infiltration. Bone marrow fat is located in the hematoma in the form of large drops. The bone marrow of fragments is impregnated with blood, as well as some Haversian canals. There is a pronounced traumatic edema. The vessels are full-blooded and hyperemic both in the central and peripheral fragments. ${ }^{11}$ According to our data, in the MRI study, morphological changes were characterized by a slight increase in the intensity of the signal from the bone marrow and a high signal from the formed hematoma. These changes are the same regardless of the age group of children and the location of the fracture. In the acute period of trauma (the first day), a narrow line of perifocal increase in signal intensity is due to microhemorrhagia, as a result of traumatic damage to the integrity of bone trabeculae. Changes in signal intensity are caused by concussive changes in the form of subcortical edema. ${ }^{12}$

On the $7-10^{\text {th }}$ day, the mesenchymal cells proliferate and grow into the blood bundle between the fragments at the fracture boundary. The hematoma is organized with the preservation of only a narrow gap between the fragments of the compact plate, which is filled with tissue detritus. ${ }^{11}$ Cellular infiltration is preserved. Preliminary connective tissue corn is formed. At this stage, it consists of young mesenchymal cells located between the vascular loops, i. E. is identical in its structure of granulation tissue.

The space between the beams and the vessels is filled with a loose fibrous connective tissue. The newly formed bony beams are always 
connected with the old bone (the edges of the fragments). At the edges of bone fragments, the granulation tissue proliferates with subsequent osteogenesis. Vessels and mesenchymal cells grow into the Havers canals. $^{11}$

According to own research, there is a progressive increase in the intensity of the signal from the bone perifocal fracture line, which indicates the activity of the process. The structure of the MR signal from the hematoma becomes non-uniform, with areas of weak signal intensity reduction. These changes are also the same regardless of the age group of children and the location of the fracture. After the fracture, during the first week, active proliferation of cells begins in the deep layer of the periosteum, the fracture zone. The emerging mass of new cells formed in the surface zone exceeds that observed on the part of the endostom. As a result of this mechanism, skeletal debris is formed at the ends of the fragments.

On the $20^{\text {th }}$ day, according to the literature, there is a fully formed pre-bone callus, represented by a coarse-fiber bone, which at most sites connects both fragments. In some cases, you can observe the initial signs of transformation of bone tissue into the final callus. In the bone marrow of each fragment, new bone trabeculae develop. In cases when the cartilage tissue was formed during the healing of the fracture, cartilage resorption occurs at this stage, calcification and replacement with its bone tissue - enchondral ossification. ${ }^{11}$ According to our own research, the maximum signal intensity from the bone is perifocal fracture line, probably as a manifestation of regional immobilization osteoporosis, which corresponds to the phase of stabilization of bone tissue parameters. The decrease in the size of the intermuscular hematoma, the sharp heterogeneity of its structure, as a manifestation of differentiation and partial lysis.

On the $30^{\text {th }}$ day, according to the literature, in most cases uncomplicated healing takes place with the onset of formation of lamellar bone structures. At the same time, there is no final restructuring of the callus - the outer part of it remains to some extent. From its outer layers a periosteum is formed, merging with the period of fragments. Internal corns dissolve with bone marrow repair. ${ }^{11}$

According to our study, in a group of children with fractures of the forearm bones, the dynamics to a decrease in the intensity of the signal from the bone perifocal fracture line is observed. Intermuscular hematoma is visualized as a linear non-uniform signal. In the second age group of children with fractures of the bones of the lower leg, there was no tendency to decrease the intensity of the signal from the bone. The data obtained make it possible to assess the violation of the motor regime and possibly insufficiently rigid fixation of the limb. ${ }^{13,14}$

\section{Conclusion}

Thus, comparing the data of the above study with the obtained data on the dynamics of the change in the MRI signal, the following conclusions can be drawn:

a. The alternating stages of bone tissue regeneration are accompanied by changes in signal intensity from the damaged segment of the long tubular bone.

b. MRI is highly sensitive to visualization of the edema of the bone marrow, as well as secondary changes in parasal soft tissues;

c. Visualization capabilities of MRI allowed to compare the phases of the reparative process and immobilization osteoporosis with the dynamics of signal intensity, the severity of trabecular edema;

d. The best visualization of edema was observed in T2VI mode;

e. A more prolonged edema of the bone marrow is observed in children of primary school age with a fracture of the shin bone;

f. MRI examination of the injured sigma for predicting the further reparative process most on the 21-24th and 40th -45 th days of trauma.

g. These studies allow predicting the development of osteogenesis and complications at different stages of treatment.

\section{Acknowledgements}

None.

\section{Conflict of interest}

The author declares that there is no conflict of interest.

\section{References}

1. Korzh AA, Bondarenko NS. Damage of bones and joints in children. X Prapor. 1994. 445 p.

2. Meltsin II, Vybornov D Yu, Gurevich AI, Tarasov et al. Changes in human bone tissue in space flight and some patterns and features. Osteoporosis and osteopathy. 2005;(1):2-6.

3. Slutsky LI, Dombrovskaya LE. "Orthopedics": Nauch. works of the Riga Institute of Traumatology and Orthopedics. Riga. 1976;14:183193.

4. TR Voronovich, IV Rolevich, AA Gubko. Healing of fractures of bones: experimental and clinical research. Minsk: Science and Technology. 1994. $180 \mathrm{p}$.

5. Karlov AV, Shakhov VP. External fixation systems and regulatory mechanisms of optimal biomechanics. Tomsk: SST; 2001. 408 p.

6. VA Doskin, Keller H, Muraenko NM. Morphofunctional constants of the child's organism. Moscow: Medicine; 1997: 187.

7. Tarasenko LL. Surgut State University, Medical Institute.

8. Zhu Shaocheng, Shi Dapeng, Lian Jianmin, et al. Yixueban Univ Med Sci. 2002;37(1):52-54

9. Berdyugina OV, Berdyugin KA. Immunological criteria for predicting delayed bone consolidation. Traumatology and Orthopedics of Russia. 2009;2(52):59-66.

10. Stepanchenko AP, Dolgova IV. Magnetic Resonance and X-ray Computed Tomography in the Diagnosis of Lesions of Bone Elements of the Knee Joint. Radiology Practice. 2005;(3).

11. Shevtsov VI, Dyachkova GV, Stepanov RV, et al. Qualitative and quantitative evaluation of reparative bone formation in the treatment of patients with fractures of the shin bones by the method of transosseous osteosynthesis. Medical Visualization. 2008;(1):96-101.

12. Shakirova FV. Morphodynamics of reparative bone tissue regeneration in dogs under conditions of osteosynthesis. Veterinary medicine. 2010;(3-4):98-99.

13. Korzh AM, Belous EN, Pankov Korzh AA. Reparative bone regeneration. Moscow: Medicine; 1972. 213 p.

14. Tarasenko TS. Surgut clinical traumatological hospital. 